\title{
Rupture of an aneurysm of the sinus of Valsalva into the superior vena cava
}

\author{
GC KAYE, SJ EDMONSON, JL CAPLIN, DS TUNSTALL-PEDOE \\ From the Department of Cardiology, St Bartholomew's Hospital, London
}

We present an unusual case in which rupture of a sinus of Valsalva during a septicaemic illness produced a fistula between the aorta and the superior vena cava.

\section{Case report}

A 64 year old man (caucasian), previously in good health, presented in July 1982 with a 24 hour history of rigors, abdominal pain, and joint pains. On examination he was febrile $\left(39 \cdot 5^{\circ} \mathrm{C}\right)$, with a blood pressure of $140 / 60 \mathrm{~mm} \mathrm{Hg}$. A soft apical systolic murmur was noted. The haemoglobin concentration was $13.1 \mathrm{~g} / \mathrm{dl}$ and the white cell count $8.9 \times$ $10 \%$ ( $84 \%$ neutrophils). A midstream specimen of urine grew Escherichia coli, as did one of six blood culture bottles. A diagnosis of septicaemia secondary to a urinary tract infection was made. He was treated for 10 days with oral amoxycillin, $500 \mathrm{mg}$ thrice daily, and made a rapid recovery.

In August 1982 he had a recurrence of rigors. Again he was febrile $\left(39 \cdot 5^{\circ} \mathrm{C}\right)$ and bilateral pleural friction rubs were noted as well as a right pleural effusion. The haemoglobin concentration was $12.9 \mathrm{~g} / \mathrm{dl}$ and the white cell count $16.1 \times$ $10 \%$ ( $92 \%$ neutrophils). A midstream specimen of urine was sterile but blood cultures grew $E$ coli. Investigation of the renal tract showed a small fluid filled cyst in the right kidney and some prostatic enlargement. It was considered that the recurrent renal tract infection could be attributed to the cyst or to the prostatic hypertrophy. The renal cyst was considered too small to allow aspiration. After a course of gentamicin, $80 \mathrm{mg}$ thrice daily, a transurethral prostatectomy was performed and benign prostatic tissue obtained. This was followed by further rigors and fever, which responded to intramuscular cephradine, $1 \mathrm{~g}$ thrice daily.

The patient was readmitted in December 1982 complaining of increasing dyspnoea and palpitations; he had clinical signs of fluid retention. On examination he was afebrile and atrial fibrillation was noted for the first time. The pulse was bounding and the blood pressure was 140/ $70 \mathrm{~mm} \mathrm{Hg}$. The jugular venous pressure was raised by 5 $\mathrm{cm}$. A loud, long systolic and an early diastolic murmur were heard maximally at the third left intercostal space. Bilateral basal crepitations and a small right pleural effusion were present. The liver was enlarged and nonpulsatile.

The haemoglobin concentration was $9.8 \mathrm{~g} / \mathrm{dl}$. A chest radiograph showed cardiomegaly; the electrocardiogram

Address for reprint requests: Dr G Kaye, Department of Cardiology, St Bartholomew's Hospital, London EC1A 7BE.

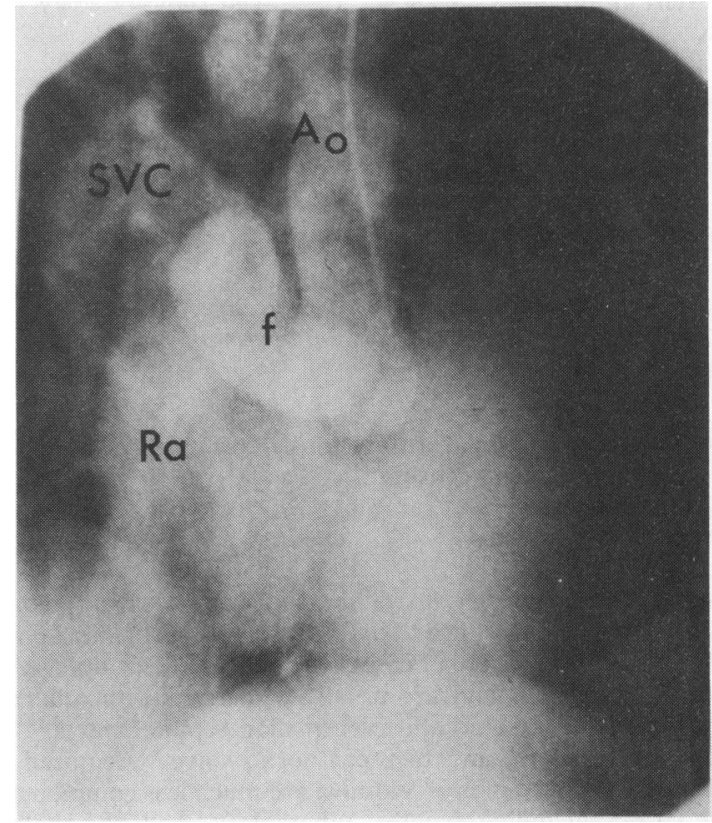

Fig 1 Angiogram in the right anterior oblique position. Ao-aorta; $f$-fistula; SVC-superior vena cava; Ra-right atrium.

was unremarkable except for atrial fibrillation. An echocardiogram showed diastolic flutter of the anterior mitral valve leaflet compatible with aortic regurgitation and left ventricular dilatation. Cardiac catheterisation showed a step up in the oxygen saturation between the high superior vena cava and the low superior vena cava from $68 \%$ to $89 \%$, with a pulmonary artery saturation of $78 \%$. An aortogram in the right anterior oblique position showed a connection between the aortic root and the superior vena cava (fig 1). Trivial aortic regurgitation was seen.

At operation (fig 2) there was a moderately large, clear pericardial effusion. With full cardiopulmonary bypass, after cross clamping, the aorta was opened. In relation to the non-coronary sinus there was a defect $3 \mathrm{~cm}$ in diameter, the roof of which communicated with the medial wall of the superior vena cava via a smooth walled sinus. The aortic valve was normal. The hole in the superior vena cava was closed with interrupted sutures. The defect in the aorta was too large to be closed directly, necessitating replacement above the coronary ostia with a Dacron tube graft. The aortic wall grew Streptococcus faecium and anti- 


\section{ROOT OF AORTA OPENED}

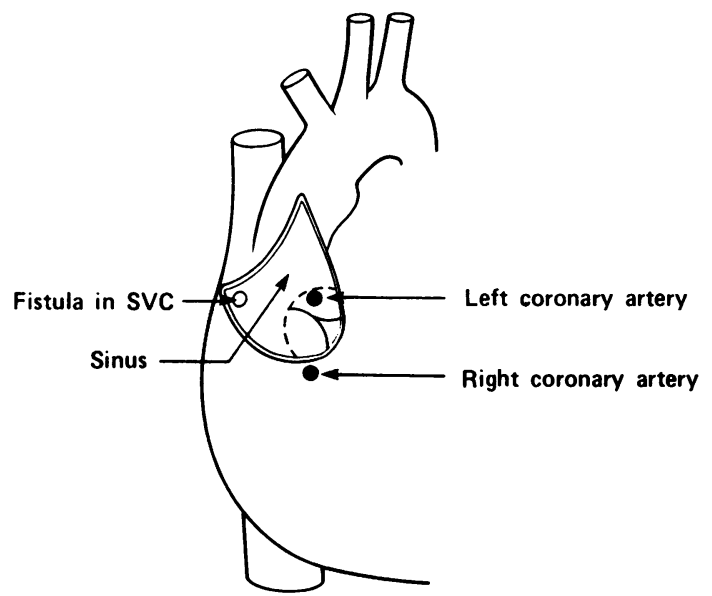

Fig 2 Diagram of defects found at operation. SVC-superior vena cava.

biotics were continued intravenously for six weeks. The patient is now fully recovered.

\section{Discussion}

Conventionally aneurysms of the sinus of Valsalva have been thought of as congenital defects in the aortic media.' They account for $3.5 \%$ of cases of congenital heart disease treated surgically ${ }^{2}$ and are usually associated with other congenital lesions, notably ventricular septal defects, ${ }^{34}$ aortic coarctation, and bicuspid aortic valve. ${ }^{2}$ Acquired aneurysms of the sinus of Valsalva are much less common, the causes including syphilis, tuberculosis, and bacterial endocarditis. ${ }^{14}$

Aneurysms of the sinus of Valsalva more commonly arise in the right coronary sinus. ${ }^{13}$ Those arising in the non-coronary and left coronary cusps are less common and all may rupture into the right side of the heart. Without surgery survival after rupture is in the region of one year, ${ }^{35}$ although patients surviving 15 years have been reported. Cases of rupture into the left atrium, left ventricle, interventricular septum, and pulmonary infundibulum have been described. ${ }^{\circ}$

Rupture into the superior vena cava has not previously been documented. This case is of particular interest as the aneurysm arose in the non-coronary cusp, which tends to rupture into the right heart chambers. ${ }^{13}$ As the edges of the sinus were so well demarcated, a congenital defect seemed likely. $S$ faecium was isolated from the aortic wall, however, suggesting that the organism, presumably introduced during prostatectomy, may have damaged the sinus further, either producing a fistula or, less likely, opening up a small defect that was already present. Cases of bacterial endocarditis producing aorticocardiac fistulas without aneurysm formation have been noted; ${ }^{2}$ possibly the initial damage to the aorta was caused by the $E$ coli septicaemia, $S$ faecium causing the subsequent aneurysm to rupture.

\section{References}

' Sakakibara S, Konno S. Congenital aneurysm of the sinus of Valsalva: anatomy and classification. Am Heart J 1962;63:405-22.

${ }^{2}$ Conde CA, Meller J, Donoso E, Dack S. Bacterial endocarditis with ruptured sinus of Valsalva and aorticocardiac fistula. Am J Cardiol 1975;35:912-6.

${ }^{3}$ Gerbode F, Osborne JJ, Johnston JB, Keith WJ. Ruptured aneurysms of the aortic sinus. Am J Surg 1961;102:268-77.

4 Jones AM, Langley FA. Aortic sinus aneurysm. Br Heart J 1949; 11:325-41.

${ }^{5}$ Oram S, East T. Rupture of aneurysm of aortic sinus (of Valsalva) into right side of the heart. $\mathrm{Br}$ Heart $\mathrm{J} 1955 ; 17: 541-51$.

- DeSa-Neto A, Padnik MB, Desser KB, Steinhoff NG. Right sinus of Valsalva aneurysm causing a right atrial fistula following non-penetrating chest trauma. Circulation 1979;60:205-9. 